\title{
Genome-wide identification of the restorer- of-fertility-like (RFL) gene family in Brassica napus and expression analysis in Shaan2A cytoplasmic male sterility
}

Luyun Ning ${ }^{1}$, Hao Wang ${ }^{2}$, Dianrong $\mathrm{Li}^{2}$, Yonghong $\mathrm{Li}^{2}$, Kang Chen ${ }^{1}$, Hongbo Chao ${ }^{1}$, Huaixin $\mathrm{Li}^{1}$, Jianjie He${ }^{1}$ and Maoteng $\mathrm{Li}^{1,3^{*}}$ (D)

\begin{abstract}
Background: Cytoplasmic male sterility (CMS) is very important in hybrid breeding. The restorer-of-fertility (Rf) nuclear genes rescue the sterile phenotype. Most of the $R f$ genes encode pentatricopeptide repeat (PPR) proteins.

Results: We investigated the restorer-of-fertility-like (RFL) gene family in Brassica napus. A total of 53 BnRFL genes were identified. While most of the BnRFL genes were distributed on 10 of the 19 chromosomes, gene clusters were identified on chromosomes A9 and C8. The number of PPR motifs in the BnRFL proteins varied from 2 to 19, and the majority of BnRFL proteins harbored more than 10 PPR motifs. An interaction network analysis was performed to predict the interacting partners of RFL proteins. Tissue-specific expression and RNA-seq analyses between the restorer line KC01 and the sterile line Shaan2A indicated that BnRFL1, BnRFL5, BnRFL6, BnRFL8, BnRFL11, BnRFL13 and BnRFL42 located in gene clusters on chromosomes A9 and C8 were highly expressed in KC01.

Conclusions: In the present study, identification and gene expression analysis of RFL gene family in the CMS system were conducted, and seven BnRFL genes were identified as candidates for the restorer genes in Shaan2A CMS. Taken together, this method might provide new insight into the study of Rf genes in other CMS systems.
\end{abstract}

Keywords: CMS, Rf, RFL, PPR, RNA-sequencing

\section{Background}

The male sterile line was widely used in hybrid breeding, which mainly included chemical induced male sterility (CIMS), genic male sterility (GMS) and cytoplasmic male sterility (CMS) $[1,2]$. In CMS, traits are maternally inherited, primarily due to the rearrangement of mitochondrial DNA and inability to generate normal pollen [3]. The

\footnotetext{
* Correspondence: limaoteng426@hust.edu.cn

'Department of Biotechnology, College of Life Science and Technology, Huazhong University of Science and Technology, Wuhan 430074, China ${ }^{3}$ Hubei Collaborative Innovation Center for the Characteristic Resources Exploitation of Dabie Mountains, Huanggang Normal University, Huanggang 438000, China

Full list of author information is available at the end of the article
}

restorer-of-fertility $(R f)$ nuclear genes have been used to rescue the damage induced by mitochondrial DNA rearrangements. In Brassica napus, there are four major CMS systems which have been commonly used in rapeseed production: pol CMS [4], nap CMS [5], Ogu CMS [6], and Shaan2A CMS [7]. Shaan2A CMS and pol CMS are the most widely used CMS systems in B. napus [8]. What's more, in Shaan2A CMS system, the cytoplasm type of its restorer line KC01 belongs to pol CMS type [9].

The first $R f$ gene encoding a putative aldehyde dehydrogenase was cloned in the T-CMS of maize (Zea mays); the encoded protein either performs acetaldehyde detoxification or interacts with the male sterile

(c) The Author(s). 2020 Open Access This article is licensed under a Creative Commons Attribution 4.0 International License, which permits use, sharing, adaptation, distribution and reproduction in any medium or format, as long as you give appropriate credit to the original author(s) and the source, provide a link to the Creative Commons licence, and indicate if changes were made. The images or other third party material in this article are included in the article's Creative Commons licence, unless indicated otherwise in a credit line to the material. If material is not included in the article's Creative Commons licence and your intended use is not permitted by statutory regulation or exceeds the permitted use, you will need to obtain permission directly from the copyright holder. To view a copy of this licence, visit http://creativecommons.org/licenses/by/4.0/ The Creative Commons Public Domain Dedication waiver (http://creativecommons.org/publicdomain/zero/1.0/) applies to the data made available in this article, unless otherwise stated in a credit line to the data. 
mitochondrial proteins [10]. To date, many other $R f$ genes have been identified in different CMS systems. Most of these $R f$ genes encode pentatricopeptide repeat (PPR) proteins. Examples of such $R f$ genes include $R f$ PPR592 in petunia (Petunia hybrida) [11], Rfo [12] and orf687 [13] in radish (Raphanus sativus), Rf4 [14], Rf5 [15] and $R f 6$ [16] in rice (Oryza sativa) and $R f p$ [17] and $R f n$ [18] in rapeseed. The PPR proteins were first identified as tandem repeats of degenerate 35-amino-acid motifs (PPR motifs) in Arabidopsis thaliana [19] and were classified into PLS and P subfamilies, according to the PPR motif structure [20]. The PPR gene family is a large family comprising 441 members in Arabidopsis [21], 491 members in rice [22] and 626 members in poplar (Рориlus alba) [23]. Except for the PPR13 in sorghum (Sorghum bicolor), most of the RF-related PPR proteins belong to the $\mathrm{P}$ subfamily and lack the catalytic sites for RNA editing or binding [15]. Two partner proteins of the RF-related PPR proteins have been reported, including GRP162, which associates with RF5 [15], and hexokinase 6 (HXK6), which functions together with RF6 [16] to rescue CMS in rice.

To date, all reported $R f$ genes have been identified via genetic mapping, which is a time-consuming method and takes several years to narrow down the genomic region of interest. However, the concept of restorer-of-fertility-like (RFL) gene was put forward in 2011, and 212 RFL genes were identified based on BLAST searches using the $R f$ PPR592 and Rf5 sequences against the genomes of 13 different dicot and monocot species, including Arabidopsis, soybean (Glycine max) and sorghum [24]. AtRFL2, together with RNase P, regulates the processing of mitochondrial orf291 RNA [25]. AtRFL4 is needed for processing the $5^{\prime}$-end of nad4 mRNA in mitochondria [26]. AtRFL9, also known as RNA PROCESSING FACTOR 4 (RPF4), participates in the generation of extra 5' termini of ccmB transcripts in Arabidopsis [27]. These results enhanced our understanding of mitochondrial RNA processing in plants and provided novel insights into the function of RFL proteins.

In the present study, we performed BLAST searches using the sequences of Rf-PPR592 and AtRFLs against the genome of rapeseed and identified 53 BnRFL genes. Based on these 53 BnRFL genes, candidate $R f$ genes were analyzed in the Shaan2A CMS system by RNA-seq and tissue-specific expression analyses. Our data provide a strong foundation for the study of $R f$ genes in other CMS systems.

\section{Results}

\section{Identification of BnRFL genes}

A total of 53 BnRFLs were identified in this study, based on the homology with the RFL genes in Arabidopsis (AtRFL1-26) and petunia (Rf-PPR592) (Table 1). First, sequences of all 26 AtRFLs were searched in the database one at a time. Of the 26 AtRFL genes, nine showed no homologs in B. napus, including AtRFL5, AtRFL6, AtRFL9, AtRFL10, AtRFL14, AtRFL15, AtRFL16, AtRFL25 and AtRFL26. Then, using Rf-PPR592 as a reference [24], 26 BnRFLs were identified (E-value < $\left.1 \mathrm{e}^{-100}\right)$. Taken together, there should be a total of 53 $B n R F L$ genes in B. napus. We also identified two known restorer genes, BnRFL6 (Rfn) and BnRFL13 (Rfp) (previously identified in the nap and pol CMS systems) and four candidate restorer genes (BnRFL2, BnRFL10, BnRFL11 and BnRFL42; previously identified in B. napus by fine genetic mapping) [31].

The number of PPR motifs in the BnRFL proteins varied from 2 to 19, although most of the BnRFL proteins contained at least 10 PPR motifs and the average number of PPR motifs was 12 (Table 1). Approximately one-fifth of the BnRFLs showed relatively low $\mathrm{pI}(<6)$, whereas nearly half of the BnRFLs showed relatively high $\mathrm{pI}(>8)$. The molecular weight of these RFL proteins ranged from $11.7-92.4 \mathrm{kDa}$. Additionally, the GRAVY value of nearly two-fifth BnRFLs and most of the selected $R f$ genes was less than 0 , indicating that these RFL proteins were hydrophilic. Most of the BnRFLs were predicted to localize to the mitochondria, which is consistent with the subcellular localization of RF proteins (Table 1).

\section{Chromosomal location and structural analysis of BnRFL genes}

First, we downloaded the chromosomal distribution of AtRFLs from The Arabidopsis Information Resource (TAIR) (Fig. 1a). All 26 AtRFLs were located in a cluster on chromosome 1 . Of the 53 BnRFLs identified in this study, 46 BnRFLs were distributed unevenly on 10 of the 19 chromosomes, and 18 and 10 BnRFLs formed highly dense clusters on chromosomes A9 and C8, respectively. The remaining seven BnRFLs were located on the unmapped scaffold (Fig. $1 \mathrm{~b}$ and $\mathrm{c}$ ).

Next, we determined the exon-intron structure of the $B n R F L$ genes and a few known restorer genes (Additional file 1). Most of the BnRFLs were intron-less, similar to the restorer genes, such as $R f 4, R f 5$ and $R f 6$, in rice CMS line. Of the 53 BnRFL genes, 10 contained a single intron, similar to the $R f$ genes, $R f k 1, R f o b$ and $\operatorname{orf} 687$, in radish. Notably, the intron in BnRFL23 was more than 4 $\mathrm{kb}$ in length, unlike other BnRFLs.

Because PPR proteins generally contain tandem repeats of PPR motifs, we searched for the PPR motifs in the BnRFL proteins and a few known restorer proteins (Additional file 2). To investigate whether BnRFLs contained additional motifs, 53 BnRFLs and 9 known restorer proteins from other species were submitted to MEME. The results showed 20 motifs in the BnRFL proteins (Fig. 2). Interestingly, all of the identified RFL 


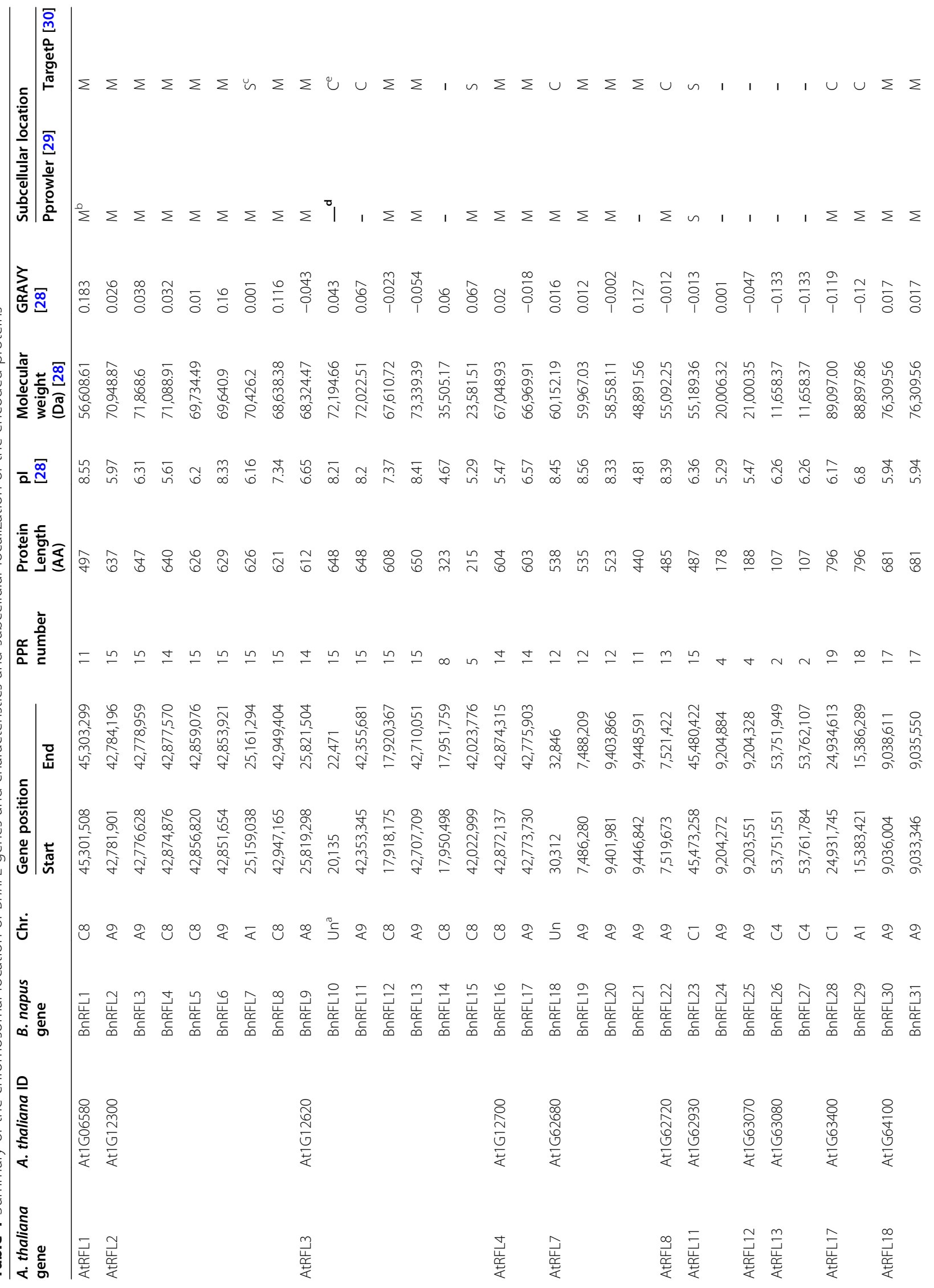




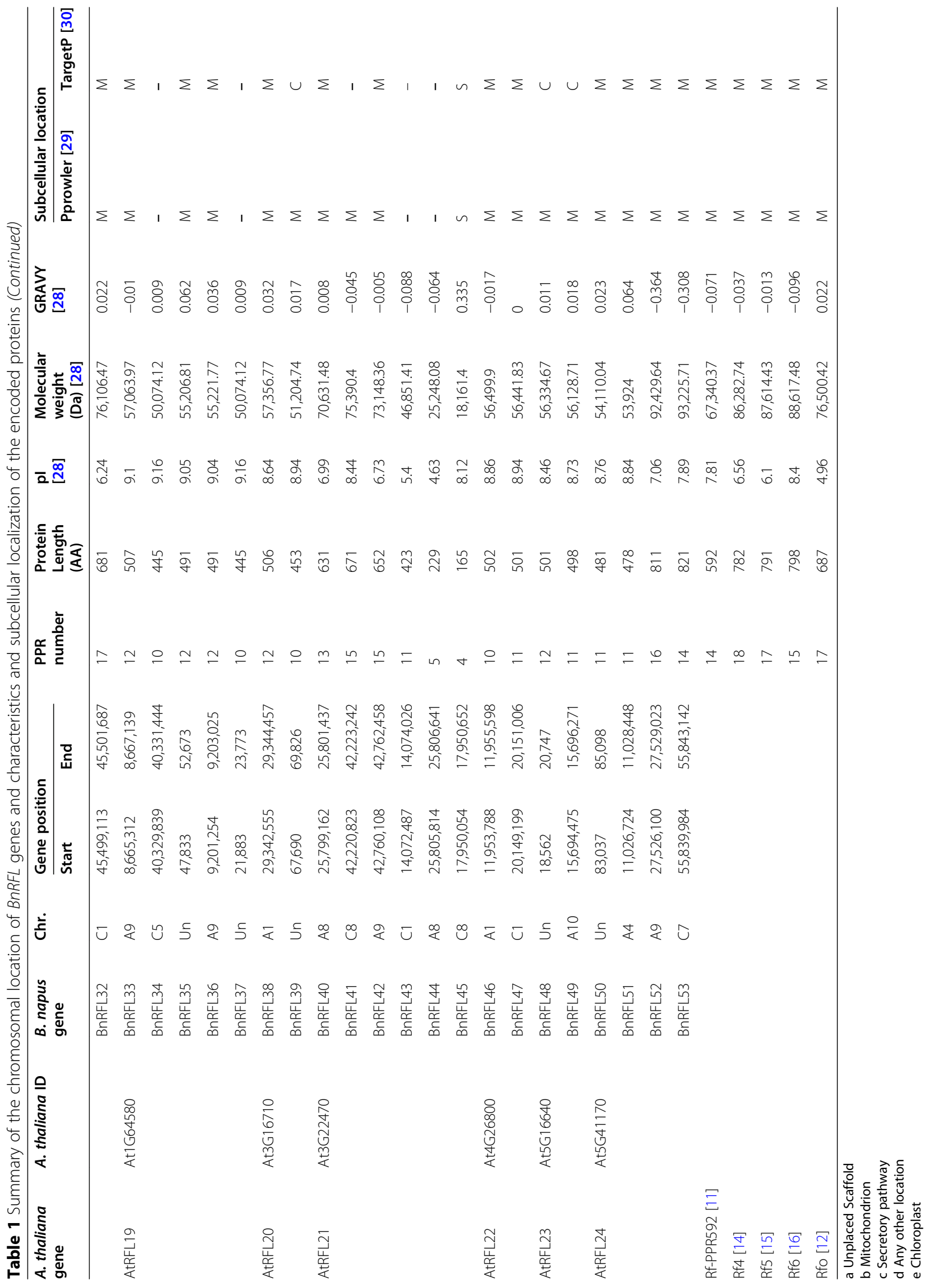




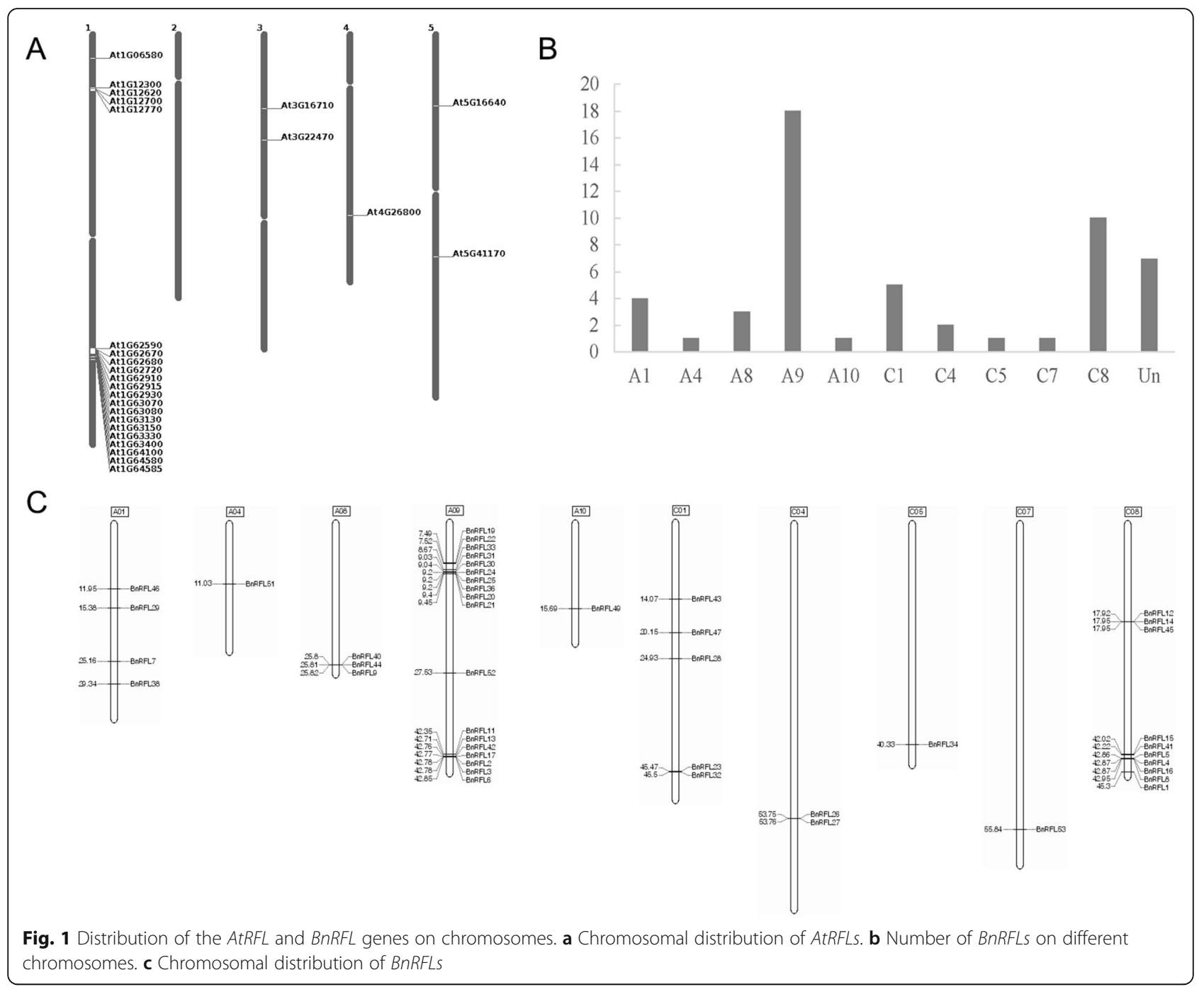

proteins contained motif 1 , which contained 80 amino acids.

\section{Phylogenetic and Syntenic analysis}

To identify the homologs of BnRFLs in different monocot and dicot species, multiple sequence alignments were performed and sequence similarity was determined. The rice RF5 protein was used for BLAST searches against the rice and maize genomes, and Rf-PPR592 was used for BLAST searches against the radish genome. An additional 16 OsRFLs (including 4 reported restorer genes), 9 ZmRFLs, and 22 RsRFLs (including 4 known restorer genes) were identified (E-value $<1 \mathrm{e}^{-100}$ ). Phylogenetic analysis revealed that RFLs mainly formed two separate clusters, and RFLs in monocot and dicot species were clustered together, respectively (Fig. 3a). Additionally, two reported restorer genes (BnRFL6 and BnRFL13) and four candidate restorer genes (BnRFL2, BnRFL10, BnRFL11 and BnRFL42) clustered together. Six additional BnRFLs (BnRFL3, BnRFL4, BnRFL5, BnRFL8, $B n R F L 15$ and BnRFL41) clustered together with the reported and candidate restorer genes. These 12 BnRFLs have been deeply investigated in the following study.

Next, we examined the synteny of BnRFLs with their homologs in Arabidopsis, B. rapa and B. oleracea (Fig. 3b). The results showed syntenic relationships between AtRFL genes on chromosome 1 and RFL genes on chromosomes BraA8, BraA9, BolC8, BnaA8, BnaA9, BnaC3, BnaC8 and BnaC9. The AtRFL gene on chromosome 3 showed synteny with RFL genes on BraA1 and BnaA1. The AtRFL genes on chromosome 4 showed synteny with RFL genes on BraA1, BolC1, BnaA1 and BnaC1, and the AtRFL genes on chromosome 5 showed synteny with RFL genes on BraA4, BnaA4, BnaC4 and BnaC9.

The Ks and Ka values indicate the evolutionary pressure on species. A $\mathrm{Ka} / \mathrm{Ks}$ ratio $<1$ indicates functional constraint, whereas $\mathrm{Ka} / \mathrm{Ks}$ ratio $>1$ indicates positive selection [32]. To explore the selection pressure on 


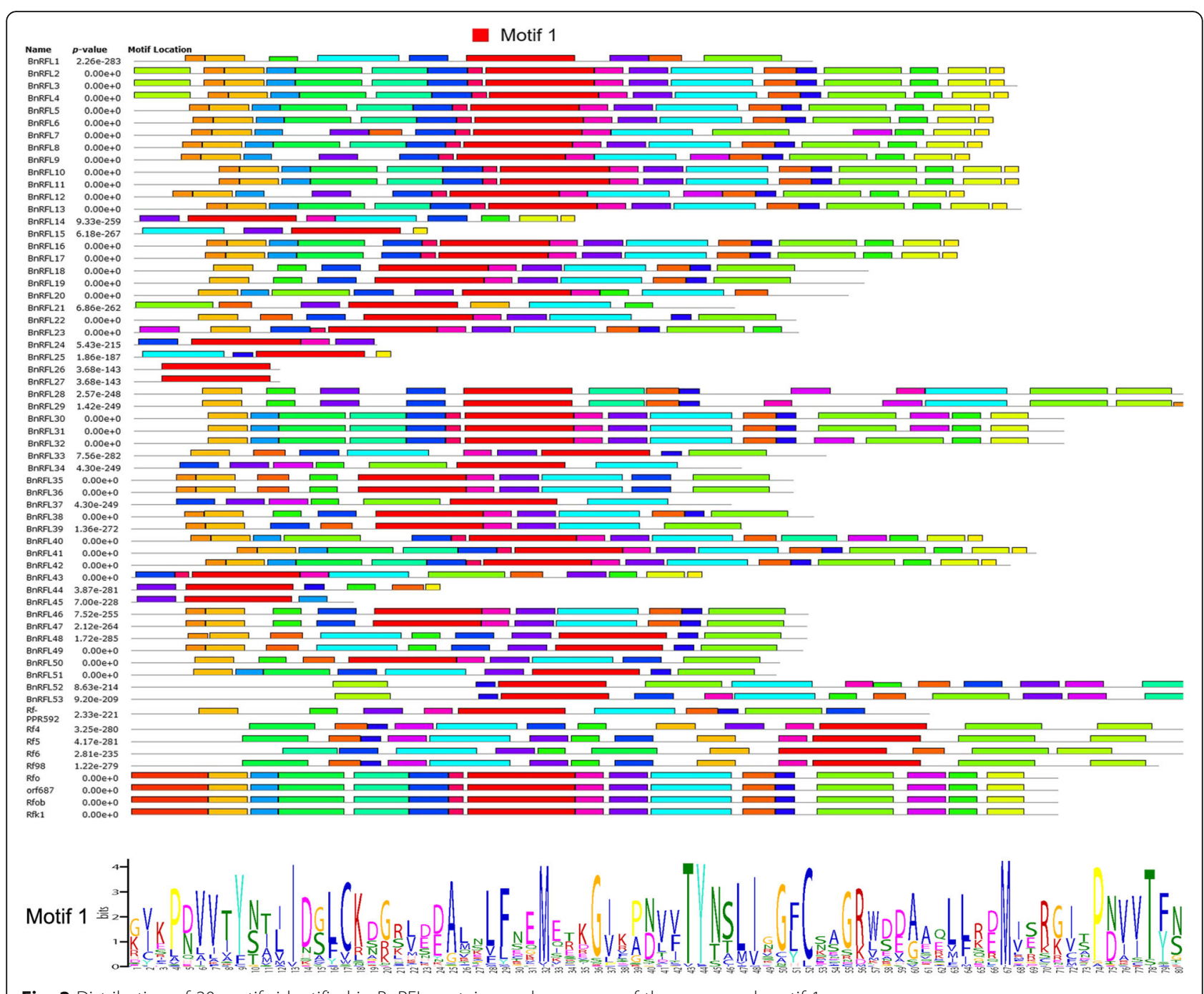

Fig. 2 Distribution of 20 motifs identified in BnRFL proteins, and sequence of the conserved motif 1

BnRFLs, we calculated the $\mathrm{Ka} / \mathrm{Ks}$ ratios (Additional file 3). All BnRFL genes showed a $\mathrm{Ka} / \mathrm{Ks}$ ratio of $0.1-0.7$. The KaKs ratio of most of the BnRFLs was relatively low (< 0.4). However, BnRFL46 and BnRFL47 showed relatively high $\mathrm{Ka} / \mathrm{Ks}$ ratios $(>0.6)$.

\section{Interaction analysis of AtRFL proteins}

Most of the RFL proteins belong to the P subfamily and need to interact with other proteins to perform RNA processing [15]. To predict the interacting partners of RFL proteins (no B. napus information in STRING database), an interaction network for AtRFLs were constructed based on STRING 10.5 and Cytoscape 3.6.1. Except AtRFL10, which did not have interaction information in the STRING database, 25 AtRFLs and their predicted partners are shown in Fig. 4 and Additional file 4. Interestingly, AtRFL11, AtRFL12 and AtRFL13 and three HXKs, including HXK1, HXK2 and HXK3, shared a common interacting protein, namely replication factor C2 (RFC2), a multi-subunit complex critical for high-speed ATP-dependent DNA synthesis [33]. No homologs of AtRFL25 were identified in B. napus. Approximately one-quarter of the BnRFL genes were homologous to AtRFL2 and AtRFL3. Further analysis revealed that AtRFL2 and AtRFL3 interact with AtRFL25, which showed interaction with the glycinerich proteins, GRP7 and GR-RBP2 (Fig. 4). Moreover, AT1G48510, SURF1, COX15 and COX11 were predicted to interact with atp6-1, AT3G48810, NAD9, $\mathrm{CCMH}$ and most of the AtRFLs.

\section{Expression analysis of BnRFL genes}

Based on the results of phylogenetic analysis, 12 BnRFL genes, which were mentioned in the phylogenetic analysis, were selected for tissue-specific expression analysis in the sterile line Shaan2A, the maintainer line Shaan2B and the restorer line $\mathrm{KC} 01$ by qRT-PCR. Although BnRFL10 and BnRFL11 were located on different 


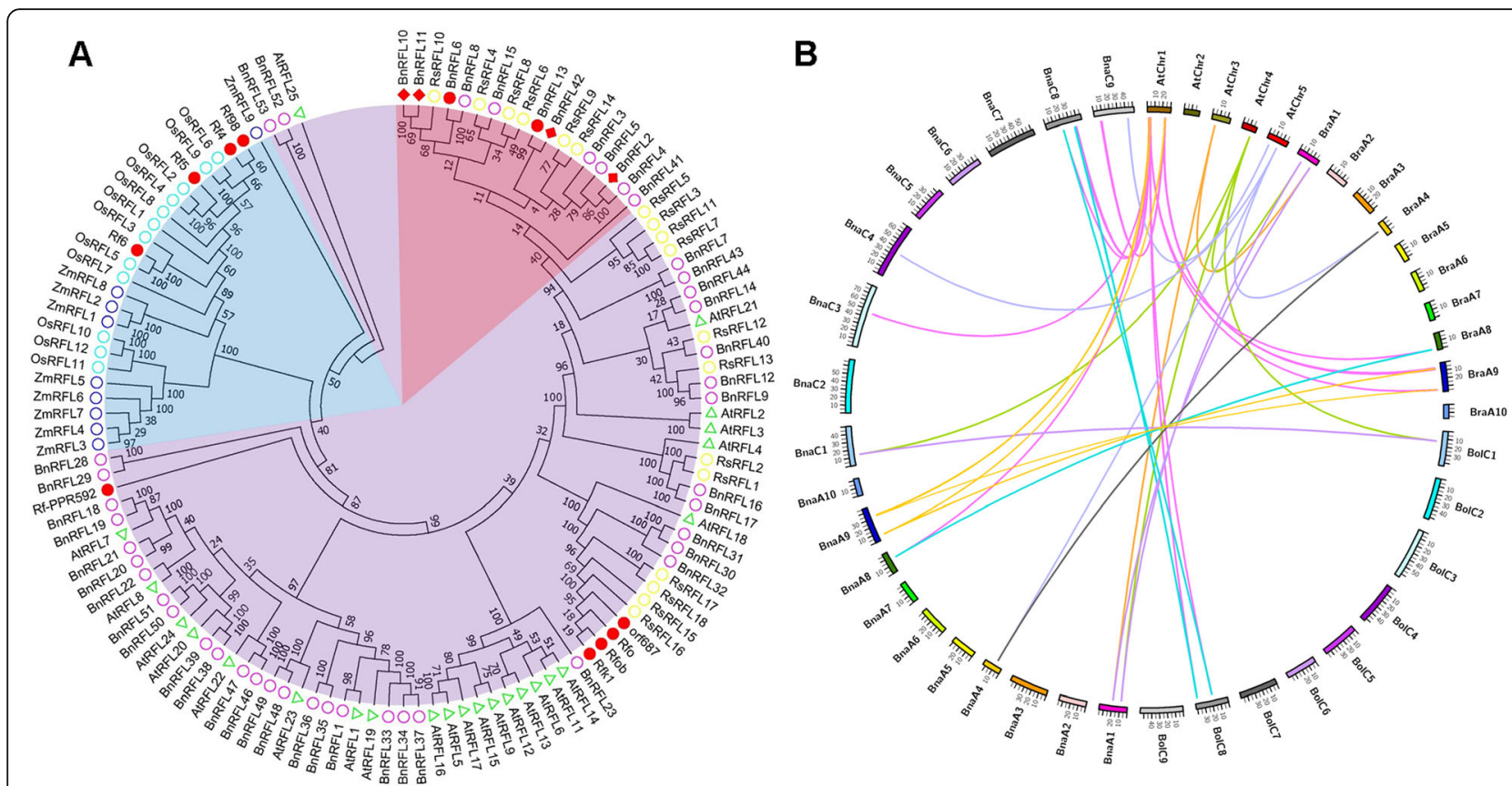

Fig. 3 Phylogenetic and syntenic analysis of RFL genes. a Phylogenetic analysis of RFLs in rapeseed (Brassica napus), Arabidopsis thaliana, rice (Oryza sativa), maize (Zea mays) and radish (Raphanus sativus). Monocot RFLs are indicated in light blue. Dicot RFLs are indicated in light purple. RFLs in the cluster of known RF proteins are indicated in pink. Red solid circles, known RF proteins; red diamonds, candidate RF proteins in a previous study. Yellow, purple, light blue and dark blue circles and green triangles represent the RFL genes in R. sativus, B. napus, O. sativa, Z. mays and $A$. thaliana, respectively. Numbers at nodes (range $0-100$ ) indicate the reliability of the corresponding branch; higher the number, higher the reliability of the branch. $\mathbf{b}$ Synteny analysis of the $R F L$ genes in A. thaliana, B. napus, B. oleracea and B. rapa. AtChr1-5, A. thaliana chromosomes 1-5; BraA1-A10, B. rapa chromosomes 1-10; BolC1-C9, B. oleracea chromosomes 1-9; BnaA1-A10, B. napus chromosomes A1-A10; BnaC1-C9, B. napus chromosomes C1-C9

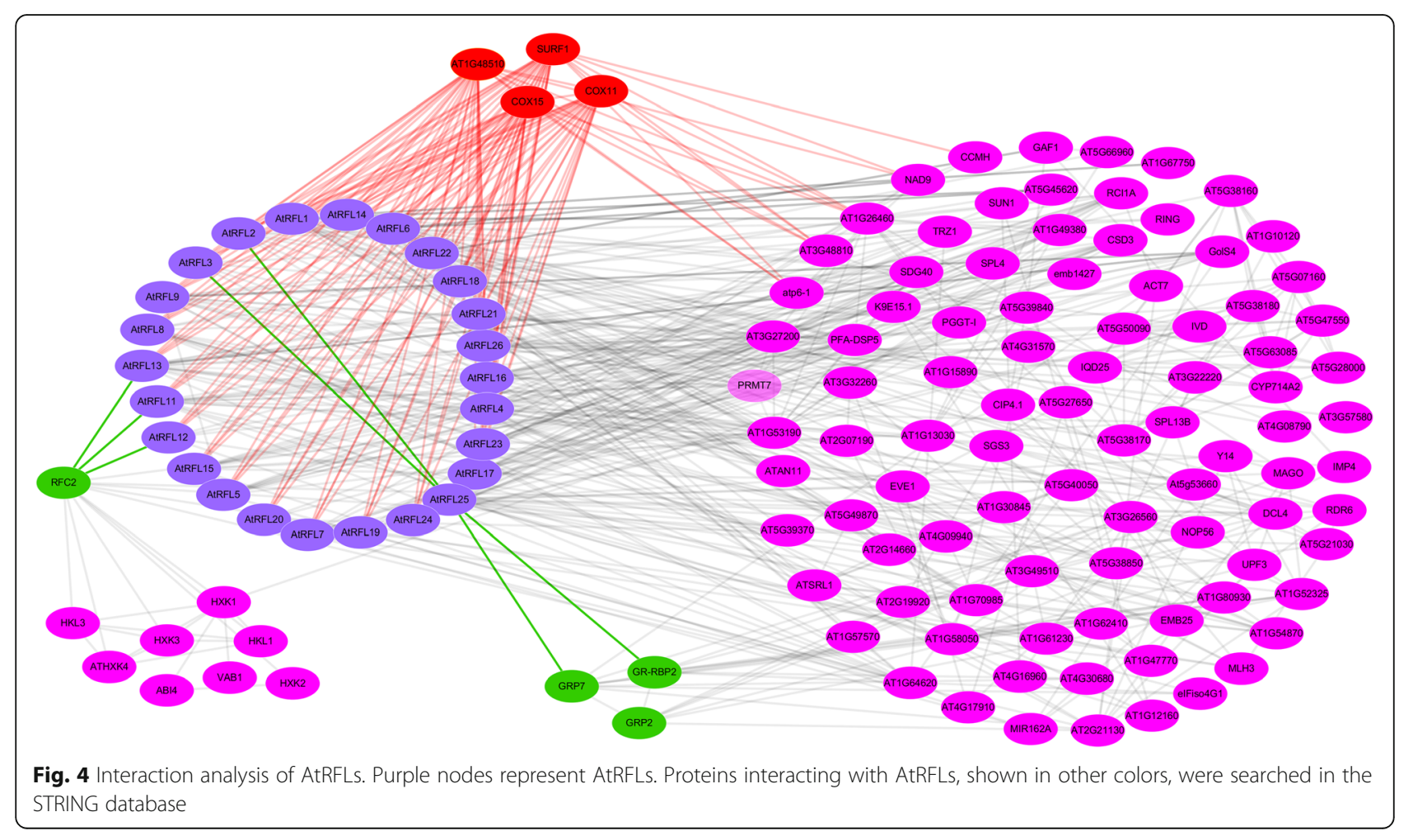


chromosomes, the coding sequence (CDSs) of these genes were highly similar (identity $=1926 / 1947$; 99\%), and it was difficult to distinguish them by qRT-PCR. Therefore, we finally analyzed the expression of 11 $B n R F L$ genes. In the restorer line, the expression of BnRFL6, BnRFL13 and BnRFL42 was lower in leaves than in the perianth (Fig. 5a, Additional file 5). The majority of the selected BnRFLs showed higher expression level in MA when compared with leaves, except for BnRFL2, BnRFL3 and BnRFL4. However, the expression of the BnRFL genes, except BnRFL41, was lower in the gynoecium and LA when compared with leaves. Compared with Shaan2A tissues, the expression of 11 BnRFL genes was higher in $\mathrm{KC} 01$ tissues, especially in MA (Fig. $5 \mathrm{~b}$, Additional file 5). What's more, the expression of these genes in Shaan2B LA was higher than those in Shaan2A (Fig. 5b, Additional file 5). However, the expression of most of these BnRFLs was lower in the gynoecium of the restorer line than in that of Shaan2A.

To compare the expression level of all 53 BnRFL genes in Shaan2A vs. KC01, three biological replicates of RNA-seq were performed using RNA isolated from young buds (YB, $<1 \mathrm{~mm}$, representing pre-meiosis) and small anthers (SA, sampled from buds $1-2 \mathrm{~mm}$ in length (representing tetrad stage to microspore release stage). A total of 320,892,232 raw sequence reads were generated, with approximately 50 million raw reads representing each tissue sample (SRA number: PRJNA511929). Additionally, to conduct comparative transcriptome analysis of the three lines in the Shaan2A CMS system, raw transcriptome reads representing the same stages of Shaan2A and Shaan2B were downloaded (SRA number: PRJNA502996). RNA-seq data analysis of Shaan2A and KC01 revealed only nine BnRFLs exhibited differential expression (Fig. 5c). These results provide important clues for analyzing the candidate restorer genes in the Shaan2A CMS system.

\section{Transcriptomic analysis between Shaan2A and KCO1}

To investigate the differences between Shaan2A and $\mathrm{KC} 01$, possibly caused by the male sterile genes and restorer genes, we also identified the differentially expressed genes (DEGs) between Shaan2B and KC01, as Shaan2A and Shaan2B share the same nuclear genetic background. Thus, common DEGs identified based on Shaan2A vs. KC01 comparison and Shaan2B vs. KC01 comparison would represent the DEGs identified between different genetic backgrounds, i.e., Shaan2A (or Shaan2B) and KC01. A total of 2980 and 8243 DEGs were identified in $\mathrm{YB}$ and SA stage, respectively, based on the comparison between Shaan2A and KC01 ( $\mid \log 2$ Ratio| > 1; Additional file 6).

\begin{tabular}{|c|c|c|c|c|c|c|c|c|c|c|c|c|c|c|c|}
\hline A & Perianth & \multicolumn{2}{|c|}{ Gynoecium } & MA & LA & \multicolumn{2}{|c|}{ C } & YB & SA & & & & & & \\
\hline$B n R F L 2$ & 0.58 & \multicolumn{2}{|c|}{-3.67} & -3.68 & -2.71 & \multicolumn{2}{|r|}{ BnRFL1 } & -2.4 & -1.72 & & & & & & \\
\hline BnRFL3 & 0.29 & \multicolumn{2}{|c|}{-0.79} & -0.53 & -1.83 & \multicolumn{2}{|r|}{ BnRFL6 } & - & -1.88 & & & & & & \\
\hline BnRFL4 & 1.22 & \multicolumn{2}{|c|}{-8.87} & -0.12 & -8.29 & \multicolumn{2}{|r|}{ BnRFL9 } & 1.42 & 1.9 & & & & & & \\
\hline BnRFL5 & 0.05 & \multicolumn{2}{|c|}{-9.19} & 1.10 & -9.63 & \multicolumn{2}{|r|}{ BnRFL10 } & -5.76 & -3.51 & & & & & & \\
\hline BnRFL6 & -3.84 & \multicolumn{2}{|c|}{-3.74} & 2.84 & -3.01 & \multicolumn{2}{|r|}{$B n R F L 13$} & -2.33 & - & & & & & & \\
\hline$B n R F L 8$ & 0.54 & \multicolumn{2}{|c|}{-0.06} & 5.39 & -0.75 & \multicolumn{2}{|r|}{ BnRFL15 } & - & -2.7 & & & & & & \\
\hline$B n R F L 10$ & 0.63 & \multicolumn{2}{|c|}{-0.22} & 3.19 & 1.00 & & $B n R F L 30$ & - & 4.77 & & & & & & \\
\hline$B n R F L 13$ & -0.84 & -0.72 & & 2.58 & 0.49 & & $B n R F L 38$ & - & -1.33 & & & & & & \\
\hline$B n R F L 15$ & 1.82 & -1.82 & & 4.39 & 1.66 & & $B n R F L 49$ & -1.51 & - & & & & & & \\
\hline$B n R F L 4 I$ & 4.62 & 3.19 & & 5.43 & 1.37 & & & & & & & & & & \\
\hline$B n R F L 42$ & -4.11 & -3.93 & & 1.68 & 3.88 & & & & & & & & & & \\
\hline B & $\begin{array}{c}2 \mathrm{~A} \\
\text { Leaf }\end{array}$ & $\begin{array}{c}2 \mathrm{~B} \\
\text { Leaf }\end{array}$ & $\begin{array}{l}\text { KC } \\
\text { Leaf }\end{array}$ & $\begin{array}{cc}2 \mathrm{~A} \\
\mathrm{f} & \text { Perianth }\end{array}$ & $\begin{array}{c}2 \mathrm{~B} \\
\text { Perianth }\end{array}$ & $\begin{array}{c}\text { KC } \\
\text { Perianth }\end{array}$ & $\begin{array}{c}2 \mathrm{~A} \\
\text { Gynoecium }\end{array}$ & $\begin{array}{c}2 \mathrm{~B} \\
\text { Gynoecium }\end{array}$ & $\begin{array}{c}\text { KC } \\
\text { Gynoecium }\end{array}$ & $\begin{array}{r}2 \mathrm{~A} \\
\mathrm{MA}\end{array}$ & $\begin{array}{r}2 \mathrm{~B} \\
\mathrm{MA}\end{array}$ & $\begin{array}{l}\mathrm{KC} \\
\mathrm{MA}\end{array}$ & $\begin{array}{l}2 \mathrm{~A} \\
\mathrm{LA}\end{array}$ & $\begin{array}{l}2 \mathrm{~B} \\
\mathrm{LA}\end{array}$ & $\begin{array}{l}\mathrm{KC} \\
\mathrm{LA}\end{array}$ \\
\hline BnRFL2 & 1.00 & 2.92 & 6.42 & 1.00 & 1.71 & 8.44 & 1.00 & 0.85 & 0.06 & 1.00 & 4.27 & 2.77 & 1.00 & 3.46 & 0.46 \\
\hline$B n R F L 3$ & 1.00 & 1.23 & 2.32 & 1.00 & 0.80 & 3.34 & 1.00 & 1.65 & 0.65 & 1.00 & 8.01 & 2.53 & 1.00 & 1.26 & 0.54 \\
\hline$B n R F L 4$ & 1.00 & 0.98 & 1.54 & 1.00 & 0.87 & 3.63 & 1.00 & 0.61 & 0.55 & 1.00 & 2.23 & 4.95 & 1.00 & 3.80 & 2.44 \\
\hline BnRFL5 & 1.00 & 1.26 & 2.42 & 1.00 & 1.44 & 4.23 & 1.00 & 1.01 & 0.66 & 1.00 & 1.33 & 3.43 & 1.00 & 2.85 & 1.00 \\
\hline$B n R F L 6$ & 1.00 & 1.24 & 5.84 & 1.00 & 0.79 & 2.11 & 1.00 & 0.69 & 0.72 & 1.00 & 1.15 & 5.03 & 1.00 & 7.71 & 7.37 \\
\hline$B n R F L 8$ & 1.00 & 2.28 & 4.96 & 1.00 & 1.31 & 2.09 & 1.00 & 1.26 & 0.98 & 1.00 & 0.50 & 3.38 & 1.00 & 2.18 & 1.12 \\
\hline BnRFL10 & 1.00 & 1.60 & 0.59 & 1.00 & 0.90 & 0.46 & 1.00 & 1.18 & 0.13 & 1.00 & 0.89 & 2.06 & 1.00 & 1.43 & 0.14 \\
\hline$B n R F L 13$ & 1.00 & 1.03 & 2.37 & 1.00 & 0.43 & 0.99 & 1.00 & 0.71 & 0.58 & 1.00 & 3.05 & 15.35 & 1.00 & 4.79 & 2.91 \\
\hline$B n R F L 15$ & 1.00 & 2.51 & 5.69 & 1.00 & 1.52 & 15.48 & 1.00 & 0.71 & 0.20 & 1.00 & 3.44 & 6.60 & 1.00 & 11.86 & 6.38 \\
\hline$B n R F L 4 I$ & 1.00 & 1.86 & 6.89 & 1.00 & 1.08 & 5.56 & 1.00 & 2.27 & 1.39 & 1.00 & 1.64 & 2.47 & 1.00 & 1.53 & 1.93 \\
\hline$B n R F L 42$ & 1.00 & 1.45 & 1.67 & 1.00 & 0.91 & 1.72 & 1.00 & 0.66 & 0.50 & 1.00 & 1.05 & 1.55 & 1.00 & 5.64 & 1.60 \\
\hline $\begin{array}{l}\text { Fig. } 5 \text { Expr } \\
\text { calculated } \\
\text { of KC01, Sh } \\
\text { the differen } \\
\text { higher expr }\end{array}$ & $\begin{array}{l}\text { ression pr } \\
\text { relative tc } \\
\text { naan2B an } \\
\text { tially exp } \\
\text { ression le }\end{array}$ & $\begin{array}{l}\text { ofiles } \\
\text { o that i } \\
\text { nd Shaa } \\
\text { ressed }\end{array}$ & 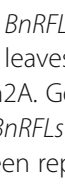 & $\begin{array}{l}\text { L genes. a EX } \\
\text { S. The } \log _{2} \\
\text { iene expressi } \\
\text { at YB and } S \\
\text { presented th }\end{array}$ & $\begin{array}{l}\text { xpressio } \\
\text {-normal } \\
\text { sion leve } \\
\text { SA stage } \\
\text { he lowe }\end{array}$ & $\begin{array}{l}\text { ofiles of } \\
\text { ratios a } \\
\text { KC01 a } \\
\text { ne expr }\end{array}$ & $\begin{array}{l}\text { f BnRFL ger } \\
\text { are shown. } \\
\text { and Shaan2 } \\
\text { ression leve } \\
\text { n levels. '-' ir }\end{array}$ & $\begin{array}{l}\text { es in differ } \\
\text { b Compara } \\
\text { B was calcu } \\
\text { Is in Shaan } \\
\text { idicates no }\end{array}$ & $\begin{array}{l}\text { rent tissue } \\
\text { ative expre } \\
\text { ulated rela } \\
72 \mathrm{~A} \text { was C }\end{array}$ & $\begin{array}{l}f K C \\
\text { on a } \\
\text { e to } \\
\text { ilate }\end{array}$ & $\begin{array}{l}\text { Ger } \\
\text { lysis } \\
\text { ose } \\
\text { relati }\end{array}$ & $\begin{array}{l}\text { expre } \\
\text { BnRF } \\
\text { Shaar } \\
\text { to th }\end{array}$ & $\begin{array}{l}\text { on in } \\
\text { enes } \\
\text { c R } \\
\text { in K }\end{array}$ & $\begin{array}{l}\text { er tis } \\
\text { iffere } \\
\text { eq ar } \\
\text { Red }\end{array}$ & $\begin{array}{l}\text { es was } \\
\text { tissues } \\
\text { sis of } \\
\text { dicated }\end{array}$ \\
\hline
\end{tabular}


Based on GO analysis, only one GO term in the molecular function category, 'sequence-specific DNA binding', was significant at YB stage (Fig. 6a). By contrast, at SA stage, 8243 DEGs identified between Shaan2A and KC01 were categorized in three main categories, including molecular function, cellular component and biological process, which were further classified into many functional sub-categories (Additional file 7). The top 30 sub- categories, including 'disaccharide metabolic process', 'regulation of RNA biosynthetic process', 'regulation of RNA metabolic process' and 'regulation of transcription, DNA-dependent', are shown in Fig. 6b.

To validate the RNA-seq data, the expression of 11 DEGs, potentially involved in anther development, was analyzed by qRT-PCR (Additional file 8 ). The results of qRT-PCR analysis for most of these DEGs at the two
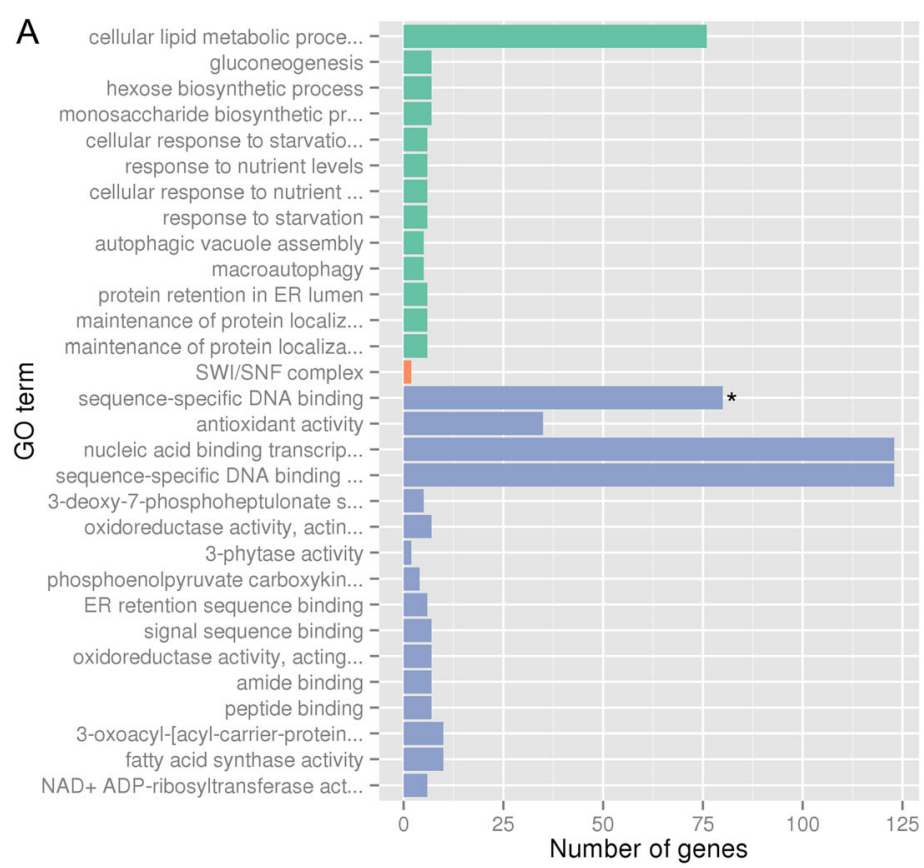

B

disaccharide metabolic process regulation of RNA biosynthetic... regulation of RNA metabolic pr... regulation of transcription, $\mathrm{D}$... photosynthesis regulation of nucleobase-conta... regulation of nitrogen compoun... biological process regulation of gene expression biosynthetic process regulation of macromolecule bi... regulation of cellular biosynt... regulation of biosynthetic pro... E regulation of cellular metabol... Ф) regulation of cellular macromo... organic cyclic compound biosyn... regulation of macromolecule me. organic substance biosynthetic... regulation of metabolic proces... photosystem cellular component oxygen evolving complex photosynthetic membrane photosystem I 2,3-bisphosphoglycerate-indepe... nucleic acid binding transcrip... sequence-specific DNA binding ...

sequence-specific DNA binding starch binding

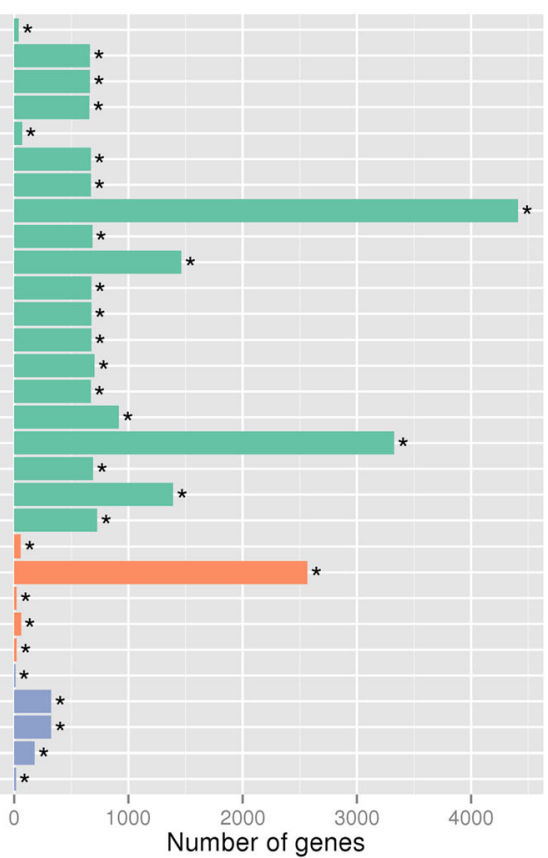

type

biological_process cellular_component molecular_function

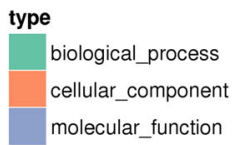

molecular_function

Fig. 6 Top 30 gene ontology (GO) sub-categories of the DEGs identified between Shaan2A and KC01. Asterisk indicates the corrected $p$-value < 0.05. a YB stage. b SA stage 
stages were consistent with those of RNA-seq analysis, indicating that the reliability of our RNA-seq data.

\section{Discussion}

Many CMS systems have been used in B. napus, including pol, nap, Ogu and Shaan2A [4-7]. To date, $R f$ genes have been identified via genetic mapping only in pol and nap CMS systems $[17,18]$. In the present study, 53 BnRFL genes were identified in the Shaan 2A CMS system. Most of these genes contained more than 10 PPR motifs, which is consistent with the previously reported restorer proteins such as Rf-PPR592 (14 PPR motifs), Rf4 (18 PPR motifs) and Rfo (17 PPR motifs) [11, 17, 24]. Moreover, most of the BnRFLs identified in this study were predicted to localize to mitochondria, similar to the known restorer genes [15-18]. Since the $R f$ genes function with the toxic chimeric genes in mitochondria to rescue male sterility [14-16], the mitochondrial localization of the proteins seems appropriate. More importantly, we also identified BnRFL6 (Rfn) and BnRFL13 $(R f p)$, previously confirmed as restorer genes in nap and pol CMS systems, respectively $[17,18]$, and four candidate restorer genes (BnRFL2, BnRFL10, BnRFL11 and $B n R F L 42)$, previously identified in $B$. napus via genetic mapping [31]. Taken together, analysis of the RFL gene family for the identification of candidate restorer genes were viable, which would also provide a new way to analysis the restorer genes in other CMS systems as one supplementary method except for the traditional genetic mapping to locate the candidate genes.

Nearly 7500 years ago, B. napus originated from the hybridization of B. rapa and B. oleracea [34], and the Brassica plants experienced the extra whole genome triplication (WGT) event when compared with Arabidopsis [35]. The Arabidopsis genome contains 26 RFL gene family members, so considering the WGT event there should be over $78 R F L$ genes in B. oleracea or $B$. rapa genome, and finally generate even more $R F L$ genes in $B$. napus. While only 53 BnRFLs were identified in the present study, which implied that nearly $50 \% R F L$ genes were lost after the WGT event.

Most of the BnRFLs were unevenly distributed on 10 of the 19 chromosomes of B. napus, while a few formed gene clusters on chromosomes A9 and C8, similar to the gene cluster in Arabidopsis (chromosome 1; Table 1, Fig. 1), rice and barley (Hordeum vulgare) [36, 37]. Gene clustering has also been observed in many other gene families, such as the $L E A$ gene family in B. napus [38] and Phyllostachys edulis [39] and laccase gene family in Citrus sinensis [40]. The LEA gene clusters on B. napus chromosomes A9 and $\mathrm{C} 4$ probably resulted from chromosomal rearrangement during the evolution of Brassica species [38]. The RFL genes on Arabidopsis chromosome 1 showed synteny with the RFL genes on
BraA9, BolC8, BnaA9 and BnaC8. Additionally, BnRFLs maintained a syntenic relationship with $R F L$ genes in $B$. rapa and $B$. oleracea, suggesting that a conserved role of $B n R F L s$ located on chromosomes A9 and C8. Moreover, AtRFL2, AtRFL4 and AtRFL9 were located within the gene cluster on Arabidopsis chromosome 1 and participated in the processing of the mitochondrial RNA [2527]. The phylogenetic analysis revealed that the BnRFLs have the closer phylogenetic relationship with AtRFLs and RsRFLs (Fig. 3), and the structural analysis showed that all of the BnRFLs and the known restorer genes in radish share a conserved motif (Fig. 2), and all BnRFL genes showed a $\mathrm{Ka} / \mathrm{Ks}$ ratio $<1$ (Additional file 3), which indicated that there was no positive selection on the $B n R F L$ genes during the evolution. What's more, BnRFL6 (Rfn) and BnRFL13 (Rfp) were located within the gene cluster on chromosome A9. These data suggest that the RFL genes within gene clusters on chromosomes $\mathrm{A} 9$ and $\mathrm{C} 8$ represent the restorer genes in the CMS system, as these likely exhibit a conserved role in mitochondrial RNA processing.

Tandem repeats of a degenerate 35-amino-acid PPR motif are the most prominent feature of the PPR family, and all of the 53 BnRFL proteins showed this trait. Although $212 R F L$ genes in 13 different species [24] and 26 RFL genes in barley [37] have been identified previously, the conserved domain of the RFL proteins has not yet been analyzed. Therefore, we investigated motifs other than PPR in the RFL proteins, revealing 20 motifs among the 53 BnRFLs and a few known RF proteins. Interestingly, all RFLs contained motif 1 , comprising 80 amino acids. We propose motif 1 as the conserved motif in the RFL protein family. This motif will serve as a reference for RFL family analysis in other species.

Because RF-related PPR proteins belong to the P subfamily and do not exhibit endonuclease activity, these proteins form functional complexes with other proteins [20]. To date, only two RFL-interacting partner proteins have been identified, including GRP162 and HXK6 in the rice CMS system $[15,16]$. In the present study, we constructed an interaction network for AtRFLs (Fig. 4). Interestingly, AtRFL11, AtRFL12 and AtRFL13 and HXKs (HXK1, HXK2 and HXK3) shared a common interacting partner, RFC2, which was critical for highspeed DNA synthesis [22], whereas AtRFL25 showed interaction with GRP7 and GR-RBP2. Moreover, AT1G48510, SURF1, COX11 and COX15 were predicted to interact with most of the AtRFLs. AT1G48510 is a surfeit locus 1 cytochrome c oxidase biogenesis protein. SURF1 is associated with cytochrome c oxidase assembly [41]. Both COX11 and COX15 are mitochondrial proteins and belong to the cytochrome c oxidase protein family [42]. COX11 likely plays a key role as a mitochondrial chaperone in the assembly of the COX complex 
and regulates pollen germination and plant growth [43]. Overall, the interaction network indicates possible partner proteins of RFL proteins in Arabidopsis. These data provide important clues for the identification of interaction factors of RF proteins in other species.

Previously, it has been shown that Rf4 is constitutively expressed in different rice organs at relatively low levels [14]. Although $R f 6$ expression is detectable in various rice tissues, it is expressed to a higher level in the panicle than in other tissues [16]. In the pol CMS system, $R f p$ shows relatively high expression in flower buds and weak expression in opening flowers, leaves, stems and roots [17]. In the phylogenetic tree, two previously reported restorer genes (BnRFL6 and BnRFL13), four candidate restorer genes (BnRFL2, BnRFL10, BnRFL11 and BnRFL42) and another six BnRFLs (BnRFL3, BnRFL4, $B n R F L 5, B n R F L 8, B n R F L 15$ and BnRFL41) clustered together, suggesting these genes as the more probable candidates of restorer genes in the rapeseed CMS system. Analysis of expression patterns revealed that most of these genes, except for BnRFL2, BnRFL3 and BnRFL4, were expressed to relatively higher levels in MA than in leaves in KC01. Additionally, these BnRFLs showed higher expression in KC01 tissues, especially MA, than in Shaan2A tissues. Expression profiling of BnRFL genes in Shaan2A vs. KC01 showed that BnRFL1, BnRFL6, BnRFL10, BnRFL13, BnRFL15, BnRFL38 and BnRFL49 were down-regulated in Shaan2A. However, BnRFL15 only harbored five PPR motifs, which was much lower than the number of PPR motifs in the known RF proteins. While BnRFL38 and BnRFL49 are located on chromosomes A1 and A10, respectively, BnRFL1, BnRFL5 and BnRFL8, BnRFL6, BnRFL11, BnRFL13 and BnRFL42 are located in gene clusters on chromosomes C8 and A9. Interestingly, almost all of the known rice $R f$ genes are located in the RFL gene cluster on chromosome 10 [36]. These data suggest BnRFL1, BnRFL5, BnRFL6, BnRFL8, BnRFL11, BnRFL13 and BnRFL42 as the more likely candidates of restorer genes in the Shaan2A CMS system. In O. sativa, RF6 with a characteristic duplication of PPR motifs in the restorer line of Honglian CMS can restore sterility, while the duplicated motifs are absent in rf6 of sterile line [16]. In the next steps, we will clone these candidate restorer genes in the restorer line and sterile line of Shaan2A CMS respectively, and compare the difference of sequences between them, for we wonder if there is the similar motif difference between these candidate restorer genes. Then we will narrow down the list of candidate genes, and conduct the transgenic work in sterile line to investigate their function.

Furthermore, DEGs identified in small anthers of Shaan2A vs. KC01 were annotated as involved in the 'regulation of RNA biosynthetic process', 'regulation of RNA metabolic process' and 'regulation of transcription,
DNA-dependent'. The RF-related PPR proteins interact with their partner proteins to bind or to edit RNA [15]. Here, the regulation of RNA biosynthetic, RNA metabolic process and transcription was different between the sterile line and restorer line, which might be caused by the sterile genes in Shaan2A and restorer genes in KC01. However, the detailed mechanism needs further investigation.

\section{Conclusions}

In CMS, the $R f$ nuclear genes rescue the sterile phenotype and most of the $R f$ genes encode pentatricopeptide repeat (PPR) proteins. In the present study, a total of 53 $B n R F L$ genes were identified in B. napus. Most of the $B n R F L$ genes were distributed on 10 of the 19 chromosomes, and gene clusters were identified on chromosomes A9 and C8. The interaction network analysis was performed to predict the interacting partners of RFL proteins. Tissue-specific expression and RNA-seq analyses between the restorer line $\mathrm{KC} 01$ and the sterile line Shaan2A indicated that BnRFL1, BnRFL5, BnRFL6, BnRFL8, BnRFL11, BnRFL13 and BnRFL42 located in gene clusters on chromosomes A9 and $\mathrm{C} 8$ were highly expressed in KC01, which suggest these seven BnRFL genes as strong candidates for the restorer genes in Shaan2A CMS. Our results would provide new insight into the study of $R f$ genes in other CMS systems.

\section{Methods \\ Plant materials}

The sterile line Shaan2A, maintainer line Shaan2B and restorer line KC01 of B. napus, gifted by Professor Dianrong $\mathrm{Li}$ at the Hybrid Rape Research Center of Shaanxi Province, were used in this study. Plants were cultivated on the experimental field of the Huazhong University of Science and Technology (Wuhan, Hubei province, China). After harvest, plant samples were immediately frozen in liquid nitrogen and stored at $-80^{\circ} \mathrm{C}$ until needed for total RNA isolation.

\section{Identification of the RFL gene family in B. napus and other species}

The $R F L$ genes were identified as described previously [24]. Briefly, AtRFL1-26 [24] and Rf-PPR592 [11] sequences were used for BLAST searches against the genome of the rapeseed cultivar 'ZS11' [44]. The sequence of rice RF5 (also known as RF1a) [15, 45] was used for BLAST searches against the genome sequences of monocots (E-value $<1 \mathrm{e}^{-100}$ ), including $O$. sativa (RGAP, http://rice.plantbiology.msu.edu/) and Z. mays [46]. The Rf-PPR592 sequence was used for BLAST searches against the genome sequences of dicots (E-value $<1 \mathrm{e}^{-100}$ ), including B. rapa [47], B. oleracea [48] and R. sativus [49]. 
The grand average of hydropathy (GRAVY) value, isoelectric point (pI) and molecular weight of RFL proteins were calculated using ExPASy (http://www.expasy.org/ tools/) [28]. The subcellular location of RFL proteins was predicted using the Protein Prowler Subcellular Localization Predictor version 1.2 (http://bioinf.scmb.uq. edu.au:8080/pprowler_webapp_1-2/) [29] and TargetP1.1 server (http://www.cbs.dtu.dk/services/TargetP/) [30].

\section{Structural analysis of RFL genes}

The exon-intron structure of BnRFL genes and a few known $R f$ genes were based on the alignments of the CDS with the corresponding genomic sequences, and the diagram was conducted using the Gene structure display server (GSDS, http://gsds.cbi.pku.edu.cn/) [50]. The PPR motifs in all BnRFL proteins and a few known RF proteins were analyzed using the NCBI Conserved Domain Search tool (http://www.ncbi.nlm.nih.gov/Structure/cdd/wrpsb.cgi) [51]. Conserved motifs in RFL proteins were analyzed using Multiple Expectation Maximization for Motif Elicitation (MEME, http://alternate.meme-suite.org/) [52].

\section{Phylogenetic and Syntenic analysis of RFL genes}

Multiple sequence alignment of the predicted amino acid sequences of BnRFLs, AtRFLs, RsRFLs, OsRFLs and ZmRFLs was performed using ClustalX [53]. A phylogenetic tree of these RFL proteins was constructed with MEGA 7 using the Neighbor Joining (NJ) method [54]. Analysis of synteny among BnRFL, AtRFL, BoRFL and $B r R F L$ genes was performed using the syntenic gene tool in the Brassica database (BRAD, http://brassicadb.org/ $\mathrm{brad} /$ ) [55]. The non-synonymous to synonymous nucleotide substitution ratio $(\mathrm{Ka} / \mathrm{Ks})$ was calculated using TBtools [56].

\section{Interaction analysis}

The interaction analysis of AtRFLs was based on the STRING 10.5 database, which included the known and predicted protein-protein interactions. First, the interaction proteins of AtRFLs were searched. After deleting the repeat proteins, the interaction network was visualized using Cytoscape 3.6.1.

\section{RNA extraction, RNA-seq and qRT-PCR analysis}

Gene expression was analyzed in various tissues of the sterile line Shaan2A and restorer line KC01 including leaves, perianths, gynoecium, medium anthers (MA) and large anthers (LA). MA were harvested from buds 2-4.5 $\mathrm{mm}$ in length and represented the uninuclear microspore stage, whereas LA were harvested from buds 4.5 $\mathrm{mm}$ in length and represented the mature pollen formation stage.
Total RNA extraction, RNA-seq analysis and qRTPCR were conducted according to our previous protocols [57], with minor modifications. Briefly, approximately $100 \mathrm{mg}$ plant samples were used for total RNA extraction using TRIzol Reagent (Invitrogen, Carlsbad, CA, USA), according to the manufacturer's instructions. Then, cDNA sequencing libraries were constructed using TruSeq ${ }^{\mathrm{Tm}}$ RNA Sample Preparation Kit (Illumina, San Diego, CA, USA). RNA-seq was performed on the Illumina NovaSeq 6000 platform. The raw data were filtered using the NGSQC toolkit (v2.2.3), and the clean reads were mapped to the reference genome of the rapeseed cultivar 'ZS11'. The differentially expressed genes (DEGs) were evaluated using DESeq2, with normalized fold-change $\geq 2$ and $p$-value $<0.05$. Gene Ontology (GO) annotation was using the Web Gene Ontology Annotation Plot (WEGO) software.

To perform qRT-PCR analysis, RNA was reverse transcribed using the TaKaRa PrimeScript ${ }^{\mathrm{Tm}}$ RT Reagent Kit with gDNA Eraser, according to the manufacturer's instructions. Actin was used as the internal reference gene [58]. The qRT-PCR experiments and transcript quantification were performed as described previously [57]. Primers used in this study are listed in Additional file 9.

\section{Supplementary Information}

Supplementary information accompanies this paper at https://doi.org/10. 1186/s12864-020-07163-Z.

Additional file 1 Exon-intron structure of the BnRFL genes and known Rf genes.

Additional file 2. Distribution of PPR motifs in the identified RFL proteins.

Additional file 3 Non-synonymous ( $\mathrm{Ka}$ ) and synonymous (Ks) nucleotide substitution rates of the coding sequence of RFL genes in A. thaliana and B. napus.

Additional file 4. List of the interaction proteins.

Additional file 5. Original data of $q R T-P C R$.

Additional file 6. RNA-seq analysis of DEGs identified between Shaan2A and $\mathrm{KCO} 1$ at YB and SA stage, respectively.

Additional file 7. List of GO sub-categories of the DEGs identified between Shaan2A and KC01.

Additional file 8. Validation of the expression of selected DEGs by qRTPCR. (A) Results of qRT-PCR analysis. (B) Results of RNA-seq analysis. The numbers indicate $\log _{2}{ }^{X}$-normalized ratios. Red indicated higher expression levels. Green represented the lower expression levels. '-' indicates no significant difference in RNA-seq data.

Additional file 9. List of primers used in this study.

\section{Abbreviations}

RFL: Restorer-of-fertility-like; CMS: Cytoplasmic male sterility; Rf: Restorer-offertility; PPR: Pentatricopeptide repeat; CIMS: Chemical induced male sterility; GMS: Genic male sterility; HXK6: Hexokinase 6; RPF4: RNA PROCESSING FACTOR 4; TAIR: The Arabidopsis Information Resource; RFC2: Replication factor C2; GRAVY: Grand average of hydropathy; CDS: Coding sequence; GSDS: Gene structure display server; NJ: Neighbor Joining; DEG: Differentially expressed gene; GO: Gene Ontology 


\section{Acknowledgements}

Not applicable.

\section{Authors' contributions}

LYN, DRL and MTL conceived and designed the study. LYN performed the experiments, analyzed the experimental data and wrote the manuscript. LYN, $H W, Y H L$ and KC participated in the identification, chromosomal location and structural analysis of BnRFL genes. $\mathrm{LYN}, \mathrm{HBC}, \mathrm{HXL}$ and JJH contributed to the phylogenetic analysis, syntenic analysis and interaction analysis. MTL reviewed the manuscript. All authors reviewed and approved the final manuscript.

\section{Funding}

This work was supported by the National Natural Science Foundation of China (31871656), the National Key Research Project of China (2016YFD0101200) and New Century Talents Support Program of the Ministry of Education of China (NCET110172).

\section{Availability of data and materials}

Raw RNA-seq data of KCO1 were submitted to the NCBI Sequence Read Archive (SRA) database under the accession number PRJNA511929. Raw RNA-Seq data of Shaan2A and Shaan2B (accession no. PRJNA502996) were downloaded from the NCBI SRA database [59]. The reference genome of the rapeseed cultivar 'ZS11', B. rapa, B. oleracea, R. sativus and Z. mays are available at NCBI under the project ID of PRJNA394926, PRJNA249065, PRJNA59981, PRJNA293438, PRJNA344915, PRJNA655717 and PRJEB32225 respectively [41, 43-46]. The reference genome of $O$. sativa was available at Rice Genome Annotation Project (RGAP, http://rice.plantbiology.msu.edu/). BnRFL1-53 can be found with NCBI accession numbers as LOC106420094, LOC106397711, LOC106397817, LOC106412080, LOC106412541, LOC106397421, LOC106350729, LOC111208528, LOC106382383, LOC106380919, LOC106369154, LOC106362038, LOC106401178, LOC106359321, LOC111208839, LOC106412542, LOC106400043, LOC106436889, LOC106368851, LOC106348977, LOC106367812, LOC106368854, LOC106395610, LOC106362947, LOC106377687, LOC106448592, LOC106448594, LOC106373934, LOC106360986, LOC106450684, LOC106450694, LOC106390267, LOC106366458, LOC106358569, LOC106420242, LOC106450895, LOC111211867, LOC106390802, LOC 106437800, LOC106382376, LOC106416119, LOC106397756, LOC106423886, LOC106378791, LOC111208626, LOC106444978, LOC106445419, LOC106432155, LOC106371992, LOC106435274, LOC106446207, LOC106367284, LOC106411529 respectively. The GRAVY value, pl and molecular weight of RFL proteins were calculated using ExPASy (http://www.expasy.org/tools/) [28]. The subcellular location of RFL proteins was predicted using the Protein Prowler Subcellular Localization Predictor version 1.2 (http://bioinf.scmb.uq.edu.au:8080/pprowler_ webapp_1-2/ [29] and TargetP1.1 server (http://www.cbs.dtu.dk/services/TargetP/) [30] respectively.

\section{Ethics approval and consent to participate}

Not applicable.

\section{Consent for publication}

Not applicable.

\section{Competing interests}

The authors declare that they have no competing interests.

\section{Author details}

'Department of Biotechnology, College of Life Science and Technology, Huazhong University of Science and Technology, Wuhan 430074, China. ${ }^{2}$ Hybrid Rape Research Center of Shaanxi Province, Shaanxi Rapeseed Branch of National Centre for Oil Crops Genetic Improvement, Yangling 712100, China. ${ }^{3}$ Hubei Collaborative Innovation Center for the Characteristic Resources Exploitation of Dabie Mountains, Huanggang Normal University, Huanggang 438000, China.

\section{Received: 30 December 2019 Accepted: 20 October 2020}

\section{Published online: 04 November 2020}

\section{References}

1. Fu TD. Breeding and utilization of rapeseed hybrid. Hubei Sci Technol. 2000: 167-9 (in Chinese).
2. Chen L, Liu YG. Male sterility and fertility restoration in crops. Annu Rev Plant Biol. 2014;65:579-606.

3. Horn R, Gupta KJ, Colombo N. Mitochondrion role in molecular basis of cytoplasmic male sterility. Mitochondrion. 2014;19:198-205.

4. Fu TD. Production and research of rapeseed in the people's republic of China. Eucarpia Cruciferae Newsletter. 1981;6:6-8.

5. Shiga T, Baba S. Cytoplasmic male sterility in rape plant, Brassica napus L. Jap J Breed. 1971;21:16-7 (in Japanese).

6. Ogura H. Studies on the male sterility in Japanese radish with special reference to the utilization of this sterility towards the practical raising of hybrid seed. Mem Fac AgricKagoshima Univ. 1968;6:39-78 (in Japanese).

7. Li DR. Report on three-lines breeding in Brassica napus. Shaanxi J Agricultural Sci. 1980;1:26-9 (in Chinese).

8. Fu TD. Breeding and utilization of rapeseed hybrids. Wuhan: Hubei Science and Technology Press; 1995. (in Chinese).

9. Zhao HX, Li ZJ, Hu SW, Sun GL, Chang JJ, Zhang ZH. Identification of cytoplasm types in rapeseed (Brassica napus L.) accessions by a multiplex PCR assay. Theor. Appl. Genet. 2010;121:643-50.

10. Cui X, Wise RP, Schnable PS. The rf2 nuclear restorer gene of male-sterile Tcytoplasm maize. Science. 1996;272:1334-6.

11. Bentolila S, Alfonso AA, Hanson MR. A pentatricopeptide repeat-containing gene restores fertility to cytoplasmic male-sterile plants. Proc Natl Acad Sci U S A. 2002;99:10887-92.

12. Brown GG, Formanová N, Jin H, Wargachuk R, Dendy C, Patil P, et al. The radish $\mathrm{Rfo}$ restorer gene of Ogura cytoplasmic male sterility encodes a protein with multiple pentatricopeptide repeats. Plant J. 2003;35:262-72.

13. Koizuka N, Imai R, Fujimoto H, Hayakawa T, Kimura Y, Kohno-Murase J, et al. Genetic characterization of a pentatricopeptide repeat protein gene, orf687, that restores fertility in the cytoplasmic male-sterile Kosena radish. Plant J. 2003;34:407-15

14. Tang H, Luo D, Zhou D, Zhang Q, Tian D, Zheng X, et al. The Rice restorer Rf4 for wild-abortive cytoplasmic male sterility encodes a mitochondriallocalized PPR protein that functions in reduction of WA352 transcripts. Mol Plant. 2014;7:1497-500.

15. Hu J, Wang K, Huang W, Liu G, Gao Y, Wang J, et al. The Rice Pentatricopeptide repeat protein RF5 restores fertility in Hong-Lian cytoplasmic male-sterile lines via a complex with the glycine-rich protein GRP162. Plant Cell. 2012;24:109-22.

16. Huang W, Yu C, Hu J, Wang L, Dan Z, Zhou W, et al. Pentatricopeptiderepeat family protein RF6 functions with hexokinase 6 to rescue rice cytoplasmic male sterility. Proc Natl Acad Sci U S A. 2015;112:14984-9.

17. Liu Z, Yang Z, Wang X, Li K, An H, Liu J, et al. A mitochondria-targeted PPR protein restores $\mathrm{pol}$ cytoplasmic male sterility by reducing orf224 transcript levels in oilseed rape. Mol Plant. 2016;9:1082-4.

18. Liu Z, Dong F, Wang X, Wang T, Su R, Hong D, et al. A pentatricopeptide repeat protein restores nap cytoplasmic male sterility in Brassica napus. J Exp Bot. 2017;68:4115-23.

19. Small ID, Peeters N. The PPR motif - a TPR-related motif prevalent in plant organellar proteins. Trends Biochem Sci. 2000;25:46-7.

20. Schmitz-Linneweber C, Small ID. Pentatricopeptide repeat proteins: a socket set for organelle gene expression. Trends Plant Sci. 2008;13:663-70.

21. Lurin C. Genome-wide analysis of Arabidopsis Pentatricopeptide repeat proteins reveals their essential role in organelle biogenesis. Plant Cell. 2004; 16:2089-103.

22. Chen G, Zou Y, Hu J, Ding Y. Genome-wide analysis of the rice PPR gene family and their expression profiles under different stress treatments. BMC Genomics. 2018;19:720.

23. Xing H, Fu X, Yang C, Tang X, Guo L, Li C, et al. Genome-wide investigation of pentatricopeptide repeat gene family in poplar and their expression analysis in response to biotic and abiotic stresses. Sci Rep. 2018;8:2817.

24. Fujii S, Bond CS, Small ID. Selection patterns on restorer-like genes reveal a conflict between nuclear and mitochondrial genomes throughout angiosperm evolution. Proc Natl Acad Sci U S A. 2011;108:1723-8.

25. Fujii S, Suzuki T, Giegé P, Higashiyama T, Koizuka N, Shikanai T. The restorerof-fertility-like 2 pentatricopeptide repeat protein and RNase $P$ are required for the processing of mitochondrial orf291 RNA in Arabidopsis. Plant J. 2016; 86:504-13.

26. Hölzle A, Jonietz C, Törjek O, Altmann T, Binder S, Forner J. A RESTORER OF FERTILITY-like PPR gene is required for 5 '-end processing of the nad4 mRNA in mitochondria of Arabidopsis thaliana. Plant J. 2011;65:737-44. 
27. Stoll K, Jonietz C, Schleicher S, des Francs-Small CC, Small ID, Binder S. In Arabidopsis thaliana distinct alleles encoding mitochondrial RNA PROCESSING FACTOR 4 support the generation of additional 5 ' termini of ccmB transcripts. Plant Mol Biol. 2017;93(6):659-68.

28. Gasteiger E. ExPASy: the proteomics server for in-depth protein knowledge and analysis. Nucleic Acids Res. 2003:31:3784-8.

29. Boden M, Hawkins J. Prediction of subcellular localization using sequencebiased recurrent networks. Bioinformatics. 2005;21:2279-86.

30. Emanuelsson O, Nielsen $H$, Brunak S, von Heijne G. Predicting subcellular localization of proteins based on their n-terminal amino acid sequence. J Mol Biol. 2000;300:1005-16.

31. Gaborieau L, Brown GG. Comparative genomic analysis of the compound Brassica napus Rf locus. BMC Genomics. 2016;17:834.

32. Anton N, Kateryna DM, Li WH. The KA/KS ratio test for assessing the protein-coding potential of genomic regions: An empirical and simulation study. Methods. 2001;12:198-202

33. Chen Y, Qian J, You L, Zhang X, Jiao J, Liu Y, et al. Subunit interaction differences between the replication factor $C$ complexes in Arabidopsis and rice. Front Plant Sci. 2018;9:779.

34. Chalhoub B, Denoeud F, Liu S, Parkin IA, Tang H, Wang X, et al. Early allopolyploid evolution in the post-Neolithic Brassica napus oilseed genome. Science. 2014;345:950-3.

35. Cheng F, Wu J, Wang X. Genome triplication drove the diversification of Brassica plants. Hortic Res. 2014;1:14024.

36. Melonek J, Stone JD, Small ID. Evolutionary plasticity of restorer-of-fertilitylike proteins in rice. Sci Rep. 2016;6:35152.

37. Melonek J, Zhou R, Bayer PE, Edwards D, Stein N, Small ID. High intraspecific diversity of restorer-of-fertility-like genes in barley. Plant J. 2018;97:281-95.

38. Liang Y, Xiong Z, Zheng J, Xu D, Zhu Z, Xiang J, et al. Genome-wide identification, structural analysis and new insights into late embryogenesis abundant (LEA) gene family formation pattern in Brassica napus. Sci Rep. 2016;6:2426

39. Huang Z, Zhong X, He J, Jin S, Guo H, Yu X, et al. Genome-wide identification, characterization, and stress-responsive expression profiling of genes encoding LEA (late embryogenesis abundant) proteins in Moso bamboo (Phyllostachys edulis). PLoS One. 2016;11:e165953.

40. Xu X, Zhou Y, Wang B, Ding L, Wang Y, Luo L, et al. Genome-wide identification and characterization of laccase gene family in Citrus sinensis. Gene. 2019;689:114-23.

41. Poyau A, Buchet $K$, Godinot C. Sequence conservation from human to prokaryotes of Surf1, a protein involved in cytochrome c oxidase assembly, deficient in Leigh syndrome. FEBS Lett. 1999:462:416-20.

42. Vishwakarma A, Tetali SD, Selinski J, Scheibe R, Padmasree K. Importance of the alternative oxidase (AOX) pathway in regulating cellular redox and ROS homeostasis to optimize photosynthesis during restriction of the cytochrome oxidase pathway in Arabidopsis thaliana. Ann Bot. 2015;116: 555-69.

43. Radin I, Mansilla N, Rödel G, Steinebrunner I. The Arabidopsis COX11 homolog is essential for cytochrome c oxidase activity. Front Plant Sci. 2015; 6:1091.

44. Sun F, Fan G, Hu Q, Zhou Y, Guan M, Tong C, et al. The high-quality genome of Brassica napus cultivar 'ZS11' reveals the introgression history in semi-winter morphotype. Plant J. 2017;92:452-68.

45. Wang Z, Zou Y, Li X, Zhang Q, Chen L, Wu H, et al. Cytoplasmic male sterility of Rice with Boro II cytoplasm is caused by a cytotoxic peptide and is restored by two related PPR motif genes via distinct modes of mRNA silencing. Plant Cell. 2006:18:676-87.

46. Jiao Y, Peluso P, Shi J, Liang T, Stitzer MC, Wang B, et al. Improved maize reference genome with single-molecule technologies. Nature. 2017;546: 524-7.

47. Wang $X$, Wang H, Wang J, Sun R, Wu J, Liu S, et al. The genome of the mesopolyploid crop species Brassica rapa. Nat Genet. 2011;43:1035-9.

48. Liu S, Liu Y, Yang X, Tong C, Edwards D, Parkin IAP, et al. The Brassica oleracea genome reveals the asymmetrical evolution of polyploid genomes. Nat Commun. 2014;5:3930

49. Kitashiba H, Li F, Hirakawa H, Kawanabe T, Zou Z, Hasegawa Y, et al. Draft sequences of the radish (Raphanus sativus L.), genome. DNA Res. 2014;21: 481-90.

50. Hu B, Jin J, Guo A, Zhang H, Luo J, Gao G. GSDS 2.0: an upgraded gene feature visualization server. Bioinformatics. 2015:31:1296-7.
51. Marchler-Bauer A, Bo Y, Han L, He J, Lanczycki CJ, Lu S, et al. CDD/SPARCLE: functional classification of proteins via subfamily domain architectures. Nucleic Acids Res. 2017;45:D200-3.

52. Bailey TL, Boden M, Buske FA, Frith M, Grant CE, Clementi L, et al. MEME SUITE: tools for motif discovery and searching. Nucleic Acids Res. 2009;37: W202-8.

53. Higgins DG, Sharp PM. CLUSTAL: a package for performing multiple sequence alignment on a microcomputer. Gene. 1988;73:237-44.

54. Kumar S, Stecher G, Tamura K. MEGA7: molecular evolutionary genetics analysis version 7.0 for bigger datasets. Mol Biol Evol. 2016;33:1870-4.

55. Cheng F, Wu J, Fang L, Wang X. Syntenic gene analysis between Brassica rapa and other Brassicaceae species. Front Plant Sci. 2012;3:198.

56. Chen CJ, Chen H, Zhang Y, Thomas HR, Frank MH, He Y, et al. TBtools, a toolkit for biologists integrating various HTS-data handling tools with a user-friendly interface. Mol Plant. 2020;13:1194-202.

57. Ning LY, Lin ZW, Gu JW, Gan L, Li Y, Wang H, et al. The initial deficiency of protein processing and flavonoids biosynthesis were the main mechanisms for the male sterility induced by SX-1 in Brassica napus. BMC Genomics. 2018;19:806.

58. An H, Yang Z, Yi B, Wen J, Shen J, Tu J, et al. Comparative transcript profiling of the fertile and sterile flower buds of pol CMS in B. napus. BMC Genomics. 2014;15:258.

59. Ning LY, Wang H, Li DR, Lin ZW, Li YH, Zhao WG, et al. Transcriptomic and proteomic analysis of Shaan2A cytoplasmic male sterility and its maintainer line in Brassica napus. Front Plant Sci. 2019;10:252.

\section{Publisher's Note}

Springer Nature remains neutral with regard to jurisdictional claims in published maps and institutional affiliations.
Ready to submit your research? Choose BMC and benefit from:

- fast, convenient online submission

- thorough peer review by experienced researchers in your field

- rapid publication on acceptance

- support for research data, including large and complex data types

- gold Open Access which fosters wider collaboration and increased citations

- maximum visibility for your research: over $100 \mathrm{M}$ website views per year

At $\mathrm{BMC}$, research is always in progress.

Learn more biomedcentral.com/submissions 